\title{
Microwave-Solvothermal Synthesis of Nanostructured BiOBr with Excellent Visible-Light Photocatalytic Properties
}

\author{
Shuisheng Wu, Qian Dong, Jun Wang, Qingming Jia, Yanlin Sun, \\ Shaoyun Shan, and Yaming Wang \\ Faculty of Chemical Engineering, Kunming University of Science and Technology, Kunming 650500, China \\ Correspondence should be addressed to Yaming Wang; wangymkmust@126.com
}

Received 17 September 2014; Revised 27 November 2014; Accepted 8 December 2014

Academic Editor: Shaobin Wang

Copyright (c) 2015 Shuisheng Wu et al. This is an open access article distributed under the Creative Commons Attribution License, which permits unrestricted use, distribution, and reproduction in any medium, provided the original work is properly cited.

\begin{abstract}
Novel $\mathrm{BiOBr}$ hierarchical microspheres have been successfully prepared via a facile microwave-assisted solvothermal route and used for visible-light photocatalytic degradation of RhB. The phase and morphology of the products were characterized by powder Xray diffraction (XRD), thermogravimetric analysis (TG), scanning electron microscopy (SEM), BET, and UV-vis diffuse reflectance spectra. The SEM observations displayed that $\mathrm{BiOBr}$ flower-like nanostructure assembled from nanosheets. The $\mathrm{BiOBr}$ flower-like nanostructure, with a narrow band gap $(2.63 \mathrm{eV})$, shows excellent photocatalytic activity in the degradation of RhB dye under visible-light, much higher than those of $\mathrm{BiOBr}$ nanosheet and P25 photocatalysts.
\end{abstract}

\section{Introduction}

$\mathrm{BiOBr}$ as one of the ternary compounds has aroused tremendous interest amidst scientific communities, since it can be used as photocatalysts [1-3] and as pigments in the cosmetic industry [4]. In view of the fact that the unique properties of nanomaterials are structure dependent [5-7], scientists have paid much attention to the controllable synthesis of $\mathrm{BiOBr}$ with nanosized particles such as hydrolysis process [8, 9], reverse microemulsion synthesis [10], and solvothermal process [11] and of hierarchical microspheres morphology [12-18]. By means of hydrothermal and/or solvothermal treatment at $140-180^{\circ} \mathrm{C}$ for $10-24 \mathrm{~h}$ and with or without template, $\mathrm{BiOBr}$ nanobelts [19], nanoflakes and nanoplates $[2,9]$, and microspheres and nanoflowers [12-15] have been successfully prepared.

In recent years, microwave-hydrothermal reaction has been used as an effective method for the synthesis of nanoparticles of various oxides $[20,21]$. Compared with conventional methods, microwave-hydrothermal synthesis has many distinct advantages: (1) shorter reaction time, (2) more uniform product dimensions and composition, and (3) easiness to tune compositions of the products [22, 23]. Herein, we report the successful synthesis of $\mathrm{BiOBr}$ hierarchical microspheres constructed by nanosheets through a facile and rapid microwave-assisted solvothermal route. Furthermore, the visible-light photocatalytic properties of $\mathrm{BiOBr}$ samples were studied.

\section{Experiment Section}

2.1. Synthesis. All the chemicals were of analytic purity and used without further purification. In a typical process, $2 \mathrm{mmol} \mathrm{Bi}\left(\mathrm{NO}_{3}\right)_{3} \cdot 5 \mathrm{H}_{2} \mathrm{O}$ and $2 \mathrm{mmol}$ cetyltrimethylammonium bromide (CTAB) were dissolved into $40.0 \mathrm{~mL}$ ethylene glycol solution while stirring for $10 \mathrm{~min}$ at room temperature, respectively. Then, CTAB solution was added dropwise to $\mathrm{Bi}\left(\mathrm{NO}_{3}\right)_{3} \cdot 5 \mathrm{H}_{2} \mathrm{O}$ solution in turn. After being vigorously stirred for $30 \mathrm{~min}$ at room temperature, the final clear buffy solution was transferred to a Teflon vessel of the MDS6 (Microwave Digestion/Extraction System, Shanghai Sineo Microwave Chemical Technology Co., Ltd.) with $80 \%$ volume filled. The microwave treatment was maintained at $160^{\circ} \mathrm{C}$ for various reaction time $(10,30,60$, and $120 \mathrm{~min}$, which were denoted as $\mathrm{BiOBr}-1, \mathrm{BiOBr}-2, \mathrm{BiOBr}-3$, and $\mathrm{BiOBr}-4$, resp.) under autogenous pressure and then naturally cooled to room temperature. The as-prepared yellow powders were repeatedly washed with the distilled water and ethanol three times, filtered, and dried in an oven at $60^{\circ} \mathrm{C}$. 


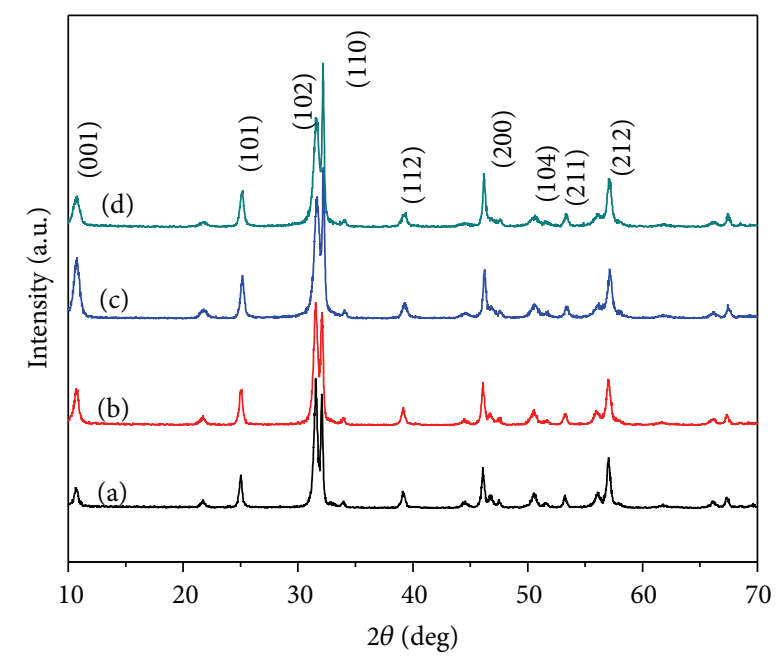

FIgURE 1: XRD pattern of BiOBr sample: (a) BiOBr-1, (b) BiOBr-2, (c) $\mathrm{BiOBr}-3$, and (d) BiOBr-4.

2.2. Characterization. The phase of as-synthesized samples was studied by X-ray diffraction (XRD) with a Bruker D8 Advance diffractometer (using $\mathrm{Cu} \mathrm{K}_{\alpha}$ radiation $(\lambda=$ $1.54056 \AA$ ) and operating at $40 \mathrm{kV}$ and $40 \mathrm{~mA}$ ). The morphology of as-prepared samples was examined by scanning electron microscopy (SEM, HITACHI S-4800) and high resolution transmission electron microscopy (HRTEM, JEM2100). $\mathrm{N}_{2}$ adsorption-desorption isotherms were measured on a Quantachrome NOVA 4000e at $77 \mathrm{~K}$. The BrunauerEmmett-Teller (BET) method was used to calculate the specific surface area. The thermal stability was investigated by a thermogravimetric analyzer (TG 209 F1, NETZSCH). The optical property was analyzed by UV-vis diffuse reflectance spectra (DRS, UV-2400PC, SHIMADZU) equipped with ISR-240A type integrating sphere.

2.3. Photocatalytic Test. The photocatalytic activities of the as-synthesized $\mathrm{BiOBr}$ flowers were evaluated in terms of the degradation of RhB in an aqueous solution. A $250 \mathrm{~W}$ highpressure mercury lamp (dominant wavelength: $365 \mathrm{~nm}$ ) used as light source was positioned inside a cylindrical vessel and surrounded by circulating water jacket for cooling. To make sure that the photocatalytic reaction was really driven by visible-light, all the UV lights with the wavelength less than $420 \mathrm{~nm}$ were removed by a glass filter (UVCUT420). $50 \mathrm{mg}$ of sample was suspended in $50 \mathrm{~mL}$ of $2 * 10^{-5} \mathrm{~mol} / \mathrm{L} \mathrm{RhB}$. The solution was continuously stirred for about $30 \mathrm{~min}$ at room temperature to ensure the establishment of an adsorptiondesorption equilibrium among the photocatalyst, $\mathrm{RhB}$, and water before irradiation with UV light from the high-pressure mercury lamp. At given time intervals, the concentration of $\mathrm{RhB}$ left in the solution was determined by the UV-vis spectroscopy (Shanghai Meipuda Instrument Co., Ltd., UV1600 ) at its characteristic wavelength of $553 \mathrm{~nm}$.

\section{Results and Discussion}

The phase and crystallographic structure of the as-obtained samples were examined by XRD. Figure 1 presents the XRD

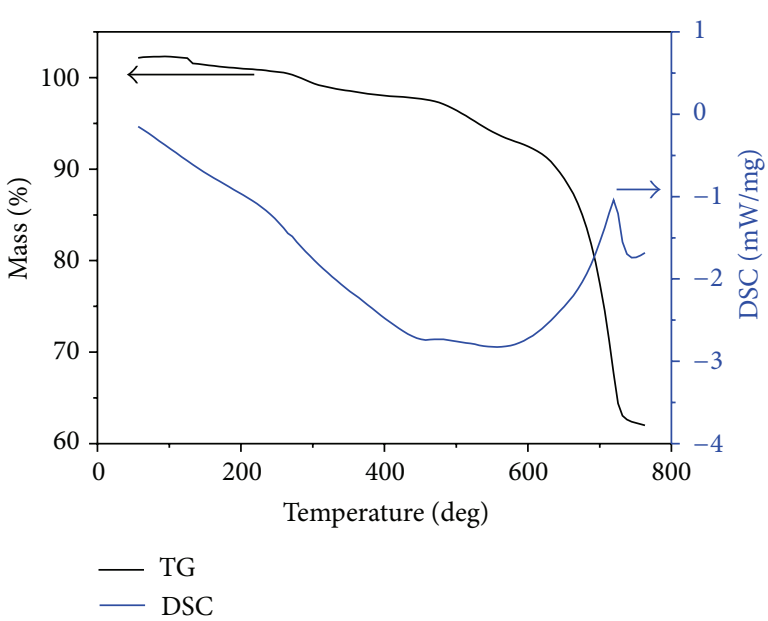

FIGURE 2: TG-DSC curves of BiOBr-4.

pattern of the as-synthesized $\mathrm{BiOBr}$ sample. The XRD pattern can clearly demonstrate that the product is well crystalline and all the diffraction peaks can be indexed unambiguously to pure tetragonal $\mathrm{BiOBr}$ with lattice constants $a=b=$ $3.926 \AA$ and $c=5.208 \AA$, well consistent with the standard PDF database (JCPDS 09-0393) [24]. The crystallite sizes of $\mathrm{BiOBr}-1, \mathrm{BiOBr}-2, \mathrm{BiOBr}-3$, and $\mathrm{BiOBr}-4$ calculated by the Scherrer equation $(D=K \lambda / \beta * \cos \theta)$ based on the principal (110) diffraction peak are $11,12.5,18.0$, and $28.0 \mathrm{~nm}$, respectively. It means that increasing the solvothermal time can enhance the crystallization of $\mathrm{BiOBr}$ catalyst. No other peaks corresponding to impurities are detected, showing that the final products purely consist of $\mathrm{BiOBr}$.

Figure 2 shows the TG and DSC curves of the BiOBr4 photocatalyst. The TG curve shows that no significant mass loss was recorded from room temperature to $600^{\circ} \mathrm{C}$. It demonstrates that the $\mathrm{BiOBr}$ sample is steady below $600^{\circ} \mathrm{C}$, so the prepared samples are of good stability and purity under the reaction temperature (room temperature) [15]. The litter mass loss may be caused by the evaporated water molecules. The mass of $\mathrm{BiOBr}$ sharply decreased from $600^{\circ} \mathrm{C}$ with an exothermic peak on the DSC curve, indicating the decomposition of $\mathrm{BiOBr}$. And remarkable exothermic phenomenon is observed at $600-800^{\circ} \mathrm{C}$, which may be caused by substance change.

The microstructure and morphology of the product were investigated by SEM. The SEM images of the resulting products are presented in Figure 3. At the initial time of $10 \mathrm{~min}$, small nanosheets in irregular shape were formed with the in-plane size of about 150-300 nm and thickness of about 20-30 $\mathrm{nm}$. Then, the nanosheets exhibited the gradual agglomeration and oriented attachment at $30 \mathrm{~min}$. As the solvothermal time prolonged to $60 \mathrm{~min}$, the integrate flowerlike microspheres were formed with larger and thinner nanosheets (about $15 \mathrm{~nm}$ thickness). Further increasing the solvothermal time to $120 \mathrm{~min}$ could achieve the larger microspheres, in which the diameter was about $1.3-1.5 \mu \mathrm{m}$ and the nanosheet thickness was about $20 \mathrm{~nm}$. Based on the above SEM morphology, the plausible mechanism of BiOBr crystal 

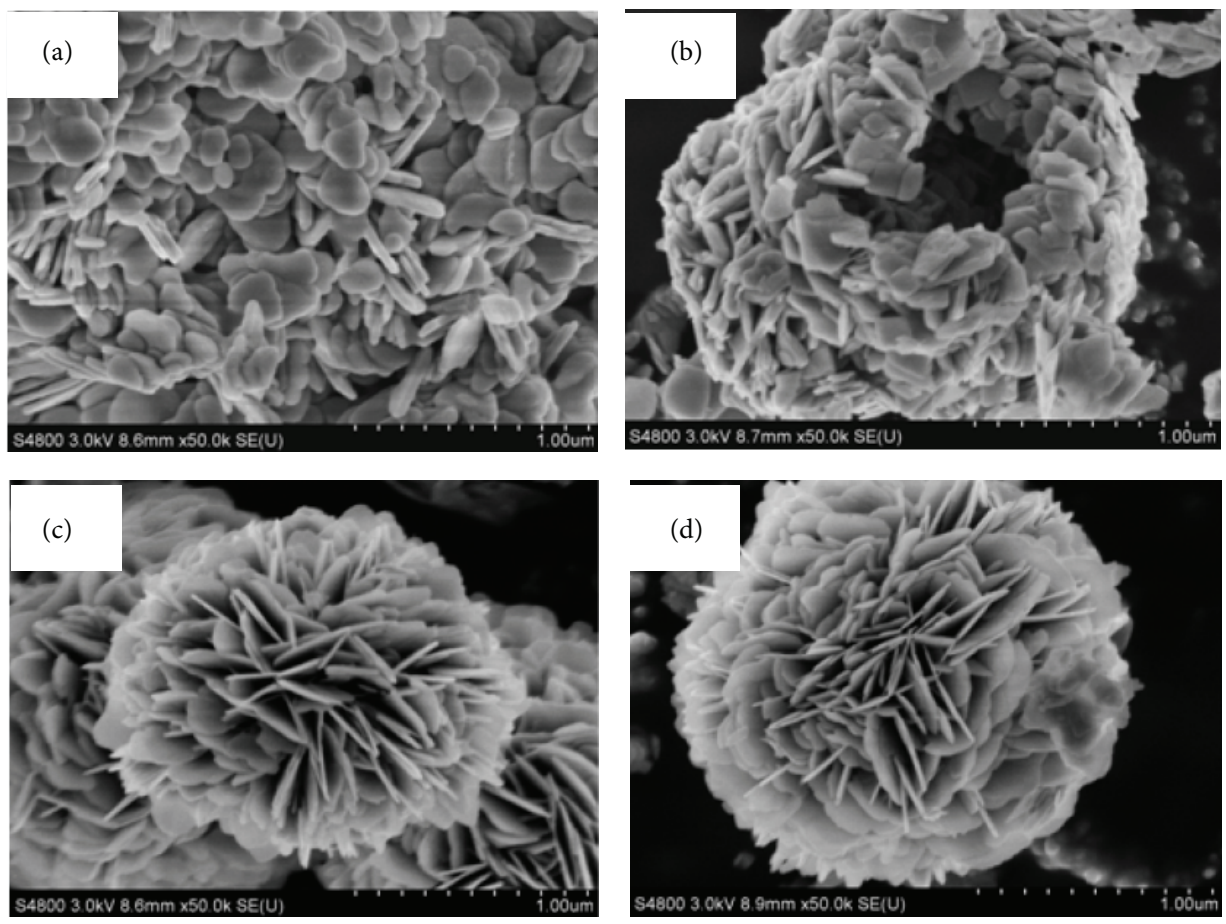

Figure 3: SEM images of BiOBr sample: (a) BiOBr-1, (b) BiOBr-2, (c) BiOBr-3, and (d) BiOBr-4.

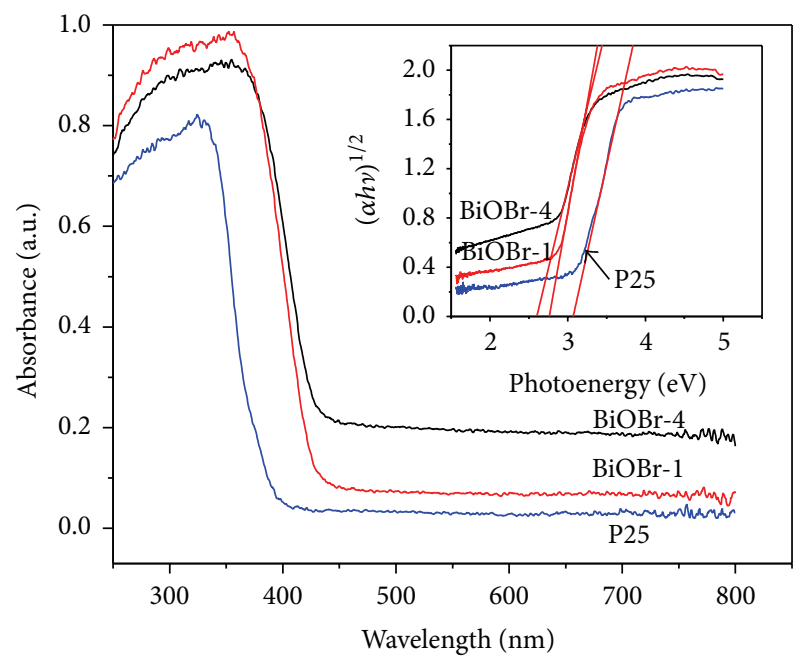

FIgURE 4: UV-vis diffuse reflectance spectra of the BiOBr sample.

growth could be proposed. Before the solvothermal reaction, the coordination occurs between ethylene glycol and $\mathrm{Bi}^{3+}$ and generates stable five-membered-ring coordination cations $\left(\mathrm{Bi}\left(\mathrm{OCH}_{2} \mathrm{CH}_{2} \mathrm{OH}\right)^{2+}\right)$, which was stable as a dense and linearly aligned structure. CTAB surfactant which acted as both template and $\mathrm{Br}$ source was self-assembled to form the lamellar structure [25]. The combination of $\mathrm{Br}^{-}$in $\mathrm{CTAB}$ lamellas with $\mathrm{Bi}\left(\mathrm{OCH}_{2} \mathrm{CH}_{2} \mathrm{OH}\right)^{2+}$ induced the formation of $\mathrm{BiOBr}$ nanosheets during the solvothermal process. With the reaction time prolonged, the $\mathrm{BiOBr}$ nanosheets were agglomerating via the oriented attachment mechanism. In the following growth stage, nanosheets grow into threedimensional (3D) flower-like hierarchical architectures built from oriented nanorods via self-assembly, driven by the minimization of the total energy of the system $[26,27]$. However, the exact formation mechanism for the eventual microstructure and morphology by interaction between the nanoparticles is still unclear at the present time.

The optical properties of the as-prepared $\mathrm{BiOBr}-4$ and $\mathrm{BiOBr}-1$ were characterized by UV-vis diffuse reflectance spectroscopy and the result was shown in Figure 4. For a crystalline semiconductor, the optical absorption near band edge follows the formula $(\alpha h \nu)^{n}=A\left(h v-E_{g}\right)$ where $\alpha, h, v, E_{g}$, and $A$ are the absorption coefficient, Plank constant, light frequency, band gap, and a constant, respectively $[2,28]$. $\mathrm{BiOBr}$ is an indirect-gap semiconductor; $n$ equals $1 / 2$. The inset of Figure 4 shows the $(\alpha h \nu)^{1 / 2}$ versus $(h \nu)$ curve of the sample. By extrapolating the straight portion of $(\alpha h \nu)^{1 / 2}$-to$(h v)$ plot to the $\alpha=0$ point, the band gap of BiOBr- 4 and $\mathrm{BiOBr}-1$ was 2.63 and $2.75 \mathrm{eV}$, which was a key factor in determining its visible-light photocatalytic activity and is close to the value reported in other literatures $[15,24]$. Furthermore, $\mathrm{BiOBr}-4$ showed higher visible-light absorption than $\mathrm{BiOBr}$ 1 , implying that more light reflection could be achieved within flower-like microspheres assembled with nanosheets.

$\mathrm{N}_{2}$ adsorption-desorption technique was carried out to investigate the surface area of the $\mathrm{BiOBr}-10$ and $\mathrm{BiOBr}-120$, and the results are given in Figure 5. Type III isotherms were obtained for $\mathrm{BiOBr}-1$ and $\mathrm{BiOBr}-4$. The BrunauerEmmett-Teller (BET) surface areas of the BiOBr-4 flower nanostructure $\left(28.3 \mathrm{~cm}^{2} / \mathrm{g}\right)$ are larger than that of $\mathrm{BiOBr}-4$ nanosheets $\left(10.4 \mathrm{~cm}^{2} / \mathrm{g}\right)$. The large surface area can be due to 


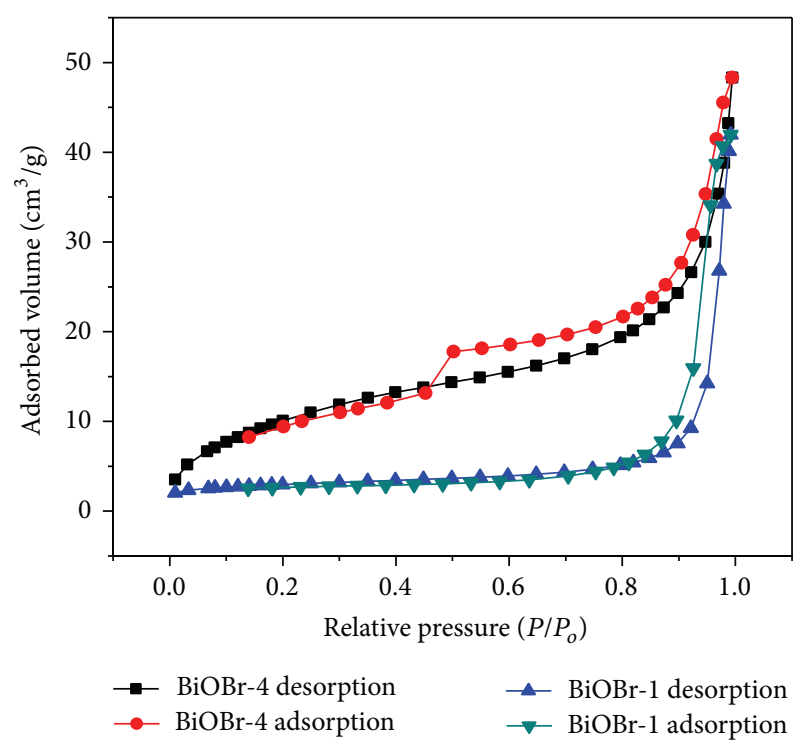

Figure 5: $\mathrm{N}_{2}$ adsorption-desorption of the $\mathrm{BiOBr}$ sample.

its flower-like structure, which is beneficial for the diffusion of reactants and intermediates during reaction.

Rhodamine B (RhB), a widely used dye, was selected as the model pollutant to evaluate the photocatalytic activity. Its characteristic absorption at about $553 \mathrm{~nm}$ has been used to monitor the photocatalytic degradation process [29]. The photodegradation of $\mathrm{RhB}$ under visible-light irradiation $(\lambda>$ $420 \mathrm{~nm}$ ) was used as a probe to evaluate the performances of different photocatalysts. Figure 6(a) shows the photocatalytic degradation of $\mathrm{RhB}$ on $\mathrm{BiOBr}$ sample, presenting the concentration changes of $\mathrm{RhB}$ during the degradation process. The degradation of $\mathrm{RhB}$ in the absence of photocatalyst is less than $5 \%$ at $90 \mathrm{~min}$, which could be neglected. About $93 \%$, close to $100 \%$ of $\mathrm{RhB}$, was degraded within $40 \mathrm{~min}$ by the sample $\mathrm{BiOBr}-4$, suggesting that the as-synthesized $\mathrm{BiOBr}$ hierarchical nanostructure possesses intrinsic photocatalytic activity. As for samples $\mathrm{BiOBr}-1$ and $\mathrm{P} 25$, the degradation rate was $75 \%$ and $33 \%$ of $\mathrm{RhB}$ within $90 \mathrm{~min}$. It is evident that the sample BiOBr-4 exhibited higher photocatalytic activity than that of samples $\mathrm{BiOBr}-1$ and P25. It could be attributed to the fact that the hierarchical flower-like morphology assembled with nanosheets in $\mathrm{BiOBr}-4$ with larger BET surface than nanosheet structure of BiOBr-4. Additionally, the hierarchical nanostructures could provide more efficient transportation of reactants in order to improve the photocatalytic activity.

The photocatalysis kinetics of the $\mathrm{RhB}$ by $\mathrm{BiOBr}$ was obtained by fitting the experimental data to pseudo-firstorder model, and the pseudo-first-order model equation can be expressed by [30],

$$
\ln \left(\frac{C}{C_{0}}\right)=-k t
$$

The $C_{0}$ and $C$ are the concentrations of organic dye in solution at times 0 and $t$, respectively, and $k$ is the pseudofirst-order rate constant. Figure 6(b) is the photocatalytic

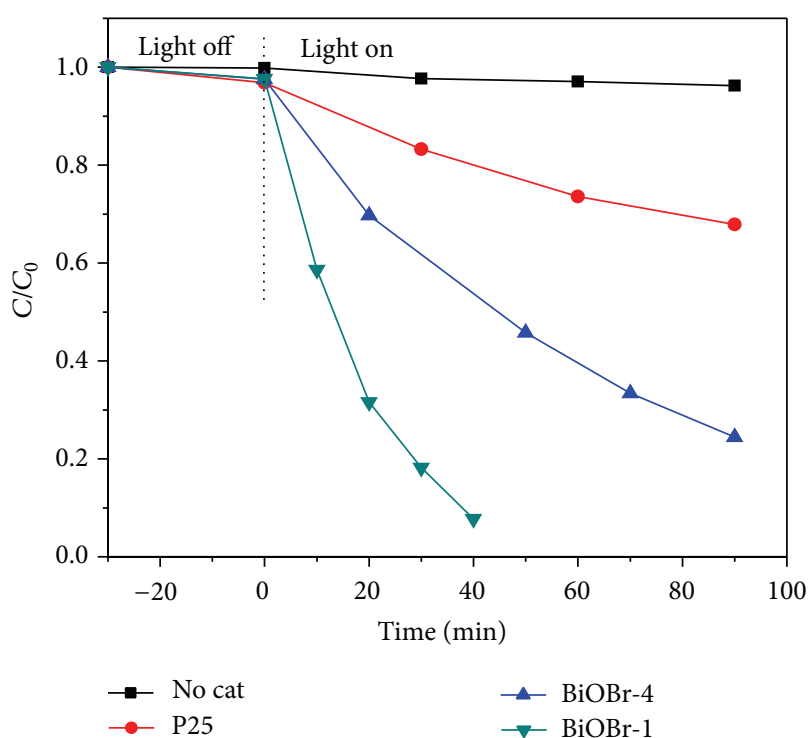

(a)

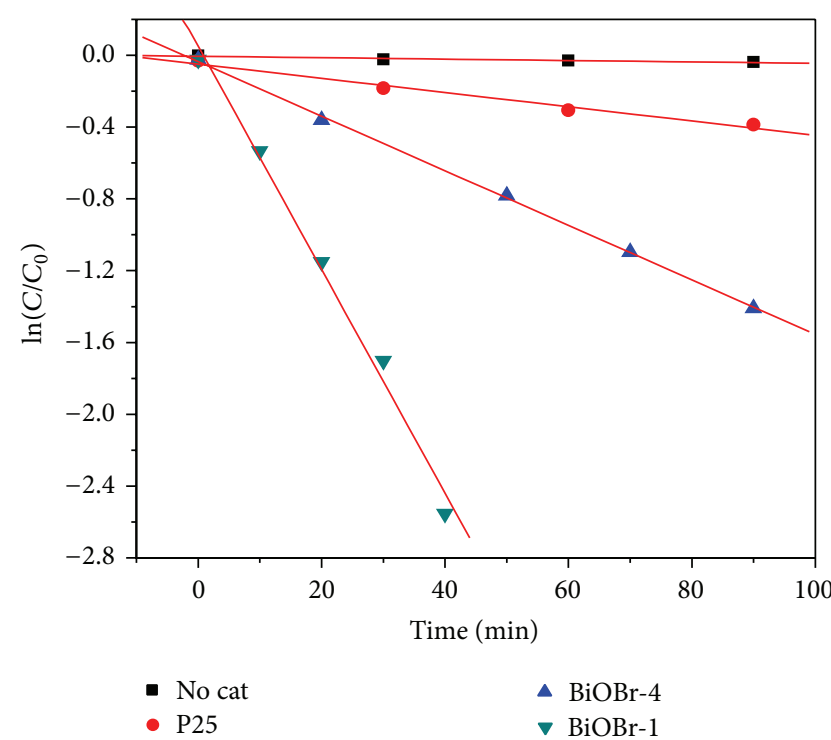

(b)

Figure 6: (a) Photodegradation efficiencies of RhB as a function of irradiation time; (b) photocatalysis kinetics of the $\mathrm{RhB}$ degradation. $C_{0}$ and $C$ referred to the initial $\mathrm{RhB}$ concentration and the $\mathrm{RhB}$ concentration determined at different reaction time, respectively.

reaction kinetics of $\mathrm{RhB}$ degradation in solution on the basis of the data plotted in Figure 6(a). As can be seen, a rather good correlation to the pseudo-first-order reaction kinetics is found. When $\mathrm{BiOBr}-4, \mathrm{BiOBr}-1$, and P25 were used as photocatalyst, the pseudo-first-order rate constants are $0.0623(R=0.99), 0.0152(R=0.99)$, and $0.0040(R=$ $0.99) \mathrm{min}^{-1}$, respectively, where the degradation rate constant of $\mathrm{BiOBr}-4$ is four times higher than $\mathrm{BiOBr}-1$ and fifteen times higher than P25. Several reasons may account for the high photocatalytic activity of the sample. First, the BET surface areas of the BiOBr-4 flower nanostructure $\left(28.3 \mathrm{~cm}^{2} / \mathrm{g}\right)$ are larger than that of $\mathrm{BiOBr}-4$ nanosheets $\left(10.4 \mathrm{~cm}^{2} / \mathrm{g}\right)$, which 
lead to more unsaturated surface coordination sites exposed to the pollution molecules in solution. This allows more efficient transport for the reactant molecules to the active sites, hence enhancing the efficiency of photocatalysis. Second, the high photocatalytic activity is not only related to the specific surface area but also associated with hierarchical structure. The rate constant of BiOBr- $4 \mathrm{BiOBr}-1$ is $0.0022 \mathrm{~min}^{-1} \cdot \mathrm{m}^{-2}$ after being normalized by the surface area, which is 1.5 times higher than that of $\mathrm{BiOBr}-1\left(0.00146 \mathrm{~min}^{-1} \cdot \mathrm{m}^{-2}\right)$; the reason may be attributed to the fact that the hierarchical microspheres composed of high surface-to-volume ratios of nanosheets are in favor of the transfer of electrons and holes and facilitate the degradation of pollution molecules.

The lifetime of photocatalyst is important for practical applications. The photodegradation of $\mathrm{RhB}$ over $\mathrm{BiOBr}-4$ has been repeated 5 times under the same reaction conditions, by repeating the centrifugation of the $\mathrm{BiOBr}$ sample after completing the photocatalysis reaction and redispersing into $50 \mathrm{~mL} \mathrm{RhB}$ solution in each run. In the lifetime test, photodegradation of $\mathrm{RhB}$ was $92.3 \%, 92.0 \%, 91.6 \%, 91.8 \%$, and $91.5 \%$, which indicates that BiOBr-4 exhibits nearly similar photocatalysis performance. It proves that $\mathrm{BiOBr}$ is stable and suitable for photocatalyst.

\section{Conclusions}

In summary, a novel $\mathrm{BiOBr}$ hierarchical microsphere has been successfully prepared via a facile microwave-assisted solvothermal route. The obtained $\mathrm{BiOBr}$ nanostructure is indirect transition semiconductors with intrinsic optical band gaps of approximately $2.63-2.75 \mathrm{eV}$ depending on the synthesis conditions. These $\mathrm{BiOBr}$ flower-like nanostructures are more active for photodegradation of $\mathrm{RhB}$ dye under visible-light irradiation. Our work provides a facile, rapid, low-cost pathway to novel $\mathrm{BiOBr}$ hierarchical nanostructures.

\section{Conflict of Interests}

The authors declare that there is no conflict of interests regarding the publication of this paper.

\section{Acknowledgments}

This work was supported by the National Natural Science Foundation (21401088), Kunming University of Science and Technology doctoral scientific research fund (KKSY201205025), and Testing and Analyzing Foundation of KMUST.

\section{References}

[1] W. D. Wang, F. Q. Huang, X. P. Lin, and J. H. Yang, "Visiblelight-responsive photocatalysts $x \mathrm{BiOBr}-(1-x) \mathrm{BiOI}$," Catalysis Communications, vol. 9, no. 1, pp. 8-12, 2008.

[2] X. Zhang, Z. H. Ai, F. L. Jia, and L. Z. Zhang, "Generalized one-pot synthesis, characterization, and photocatalytic activity of hierarchical BiOX (X $=\mathrm{Cl}, \mathrm{Br}, \mathrm{I})$ nanoplate microspheres," Journal of Physical Chemistry C, vol. 112, no. 3, pp. 747-753, 2008.
[3] J. Zhang, F. J. Shi, J. Lin et al., "Self-assembled 3-D architectures of $\mathrm{BiOBr}$ as a visible light-driven photocatalyst," Chemistry of Materials, vol. 20, no. 9, pp. 2937-2941, 2008.

[4] F. J. Maile, G. Pfaff, and P. Reynders, "Effect pigments-past, present and future," Progress in Organic Coatings, vol. 54, no. 3, pp. 150-163, 2005.

[5] Z. L. Wang and J. H. Song, "Piezoelectric nanogenerators based on zinc oxide nanowire arrays," Science, vol. 312, no. 5771, pp. 242-246, 2006.

[6] H. L. Xu and W. Z. Wang, "Template synthesis of multishelled $\mathrm{Cu}_{2} \mathrm{O}$ hollow spheres with a single-crystalline shell wall," Angewandte Chemie - International Edition, vol. 46, no. 9, pp. 1489-1492, 2007.

[7] L. Zhou, W. Wang, H. Xu, S. Sun, and M. Shang, " $\mathrm{Bi}_{2} \mathrm{O}_{3}$ hierarchical nanostructures: controllable synthesis, growth mechanism, and their application in photocatalysis," Chemistry A: European Journal, vol. 15, no. 7, pp. 1776-1782, 2009.

[8] W. Wang, F. Huang, X. Lin, and J. Yang, "Visible-lightresponsive photocatalysts $x \mathrm{BiOBr}-(1-x) \mathrm{BiOI}, "$ Catalysis Communications, vol. 9, no. 1, pp. 8-12, 2008.

[9] Z. Jiang, F. Yang, G. Yang et al., "The hydrothermal synthesis of $\mathrm{BiOBr}$ flakes for visible-light-responsive photocatalytic degradation of methyl orange," Journal of Photochemistry and Photobiology A: Chemistry, vol. 212, no. 1, pp. 8-13, 2010.

[10] J. Henle, P. Simon, A. Frenzel, S. Scholz, and S. Kaskel, "Nanosized $\mathrm{BiOX}(\mathrm{X}=\mathrm{Cl}, \mathrm{Br}, \mathrm{I})$ particles synthesized in reverse microemulsions," Chemistry of Materials, vol. 19, no. 3, pp. 366373, 2007.

[11] Z. H. Ai, W. k. Ho, S. C. Lee, and L. Z. Zhang, "Efficient photocatalytic removal of $\mathrm{NO}$ in indoor air with hierarchical bismuth oxybromide nanoplate microspheres under visible light," Environmental Science and Technology, vol. 43, no. 11, pp. 4143-4150, 2009.

[12] Y. Huo, J. Zhang, M. Miao, and Y. Jin, "Solvothermal synthesis of flower-like $\mathrm{BiOBr}$ microspheres with highly visible-light photocatalytic performances," Applied Catalysis B: Environmental, vol. 111-112, pp. 334-341, 2012.

[13] J. Xu, W. Meng, Y. Zhang, L. Li, and C. Guo, "Photocatalytic degradation of tetrabromobisphenol A by mesoporous $\mathrm{BiOBr}$ : efficacy, products and pathway," Applied Catalysis B: Environmental, vol. 107, no. 3-4, pp. 355-362, 2011.

[14] L. Zhang, X.-F. Cao, X.-T. Chen, and Z.-L. Xue, "BiOBr hierarchical microspheres: microwave-assisted solvothermal synthesis, strong adsorption and excellent photocatalytic properties," Journal of Colloid and Interface Science, vol. 354, no. 2, pp. 630636, 2011

[15] Y. C. Feng, L. Li, J. W. Li, J. F. Wang, and L. Liu, "Synthesis of mesoporous $\mathrm{BiOBr} 3 \mathrm{D}$ microspheres and their photodecomposition for toluene," Journal of Hazardous Materials, vol. 192, no. 2, pp. 538-544, 2011.

[16] G. Li, F. Qin, H. Yang, Z. Lu, H. Sun, and R. Chen, "Facile microwave synthesis of $3 \mathrm{D}$ flowerlike BiOBr nanostructures and their excellent Cr VI removal capacity," European Journal of Inorganic Chemistry, vol. 2012, no. 15, pp. 2508-2513, 2012.

[17] D. Q. Zhang, M. C. Wen, B. Jiang, G. S. Li, and J. C. Yu, "Ionothermal synthesis of hierarchical BiOBr microspheres for water treatment," Journal of Hazardous Materials, vol. 211-212, pp. 104-111, 2012.

[18] P. P. Xiao, L. L. Zhu, Y. C. Zhu, and Y. T. Qian, "Roomtemperature synthesis of $\mathrm{BiOBr}$ sub-microflowers and their photocatalytic properties," Journal of Nanoscience and Nanotechnology, vol. 12, no. 3, pp. 2008-2013, 2012. 
[19] C. F. Guo, J. Zhang, Y. Tian, and Q. Liu, "A general strategy to superstructured networks and nested self-similar networks of bismuth compounds," ACS Nano, vol. 6, no. 10, pp. 8746-8752, 2012.

[20] S. F. Liu, I. R. Abothu, and S. Komarneni, "Barium titanate ceramics prepared from conventional and microwave hydrothermal powders," Materials Letters, vol. 38, no. 5, pp. 344-350, 1999.

[21] A. Bonamartini Corradi, F. Bondioli, A. M. Ferrari, and T. Manfredini, "Synthesis and characterization of nanosized ceria powders by microwave-hydrothermal method," Materials Research Bulletin, vol. 41, no. 1, pp. 38-44, 2006.

[22] G. A. Tompsett, W. C. Conner, and K. S. Yngvesson, "Microwave synthesis of nanoporous materials," ChemPhysChem, vol. 7, no. 2, pp. 296-319, 2006.

[23] I. Bilecka, I. Djerdj, and M. Niederberger, "One-minute synthesis of crystalline binary and ternary metal oxide nanoparticles," Chemical Communications, no. 7, pp. 886-888, 2008.

[24] M. Shang, W. Z. Wang, and L. Zhang, "Preparation of BiOBr lamellar structure with high photocatalytic activity by CTAB as Br source and template," Journal of Hazardous Materials, vol. 167, no. 1-3, pp. 803-809, 2009.

[25] A. Lak, M. Mazloumi, M. Mohajerani et al., "Self-assembly of dandelion-like hydroxyapatite nanostructures via hydrothermal method," Journal of the American Ceramic Society, vol. 91, no. 10, pp. 3292-3297, 2008.

[26] L. Xu, J. Shen, C. Lu, Y. Chen, and W. Hou, "Self-assembled three-dimensional architectures of $\mathrm{Y}_{2}(\mathrm{WO} 4)_{3}$ :Eu: controlled synthesis, growth mechanism, and shape-dependent luminescence properties," Crystal Growth and Design, vol. 9, no. 7, pp. 3129-3136, 2009.

[27] L. Chen, S.-F. Yin, R. Huang, Y. Zhou, S.-L. Luo, and C.-T. Au, "Facile synthesis of $\mathrm{BiOCl}$ nano-flowers of narrow band gap and their visible-light-induced photocatalytic property," Catalysis Communications, vol. 23, pp. 54-57, 2012.

[28] S. Wu, H. Cao, S. Yin, X. Liu, and X. Zhang, "Amino acidassisted hydrothermal synthesis and photocatalysis of $\mathrm{SnO}_{2}$ nanocrystals," The Journal of Physical Chemistry C, vol. 113, no. 41, pp. 17893-17898, 2009.

[29] J.-M. Herrmann, H. Tahiri, Y. Ait-Ichou, G. Lassaletta, A. R. González-Elipe, and A. Fernandez, "Characterization and photocatalytic activity in aqueous medium of $\mathrm{TiO}_{2}$ and Ag$\mathrm{TiO}_{2}$ coatings on quartz," Applied Catalysis B: Environmental, vol. 13, no. 3-4, pp. 219-228, 1997.

[30] R. G. Chen, J. H. Bi, L. Wu, Z. H. Li, and X. Z. Fu, "Orthorhombic $\mathrm{Bi}_{2} \mathrm{GeO}_{5}$ nanobelts: synthesis, characterization, and photocatalytic properties," Crystal Growth and Design, vol. 9, no. 4, pp. 1775-1779, 2009. 

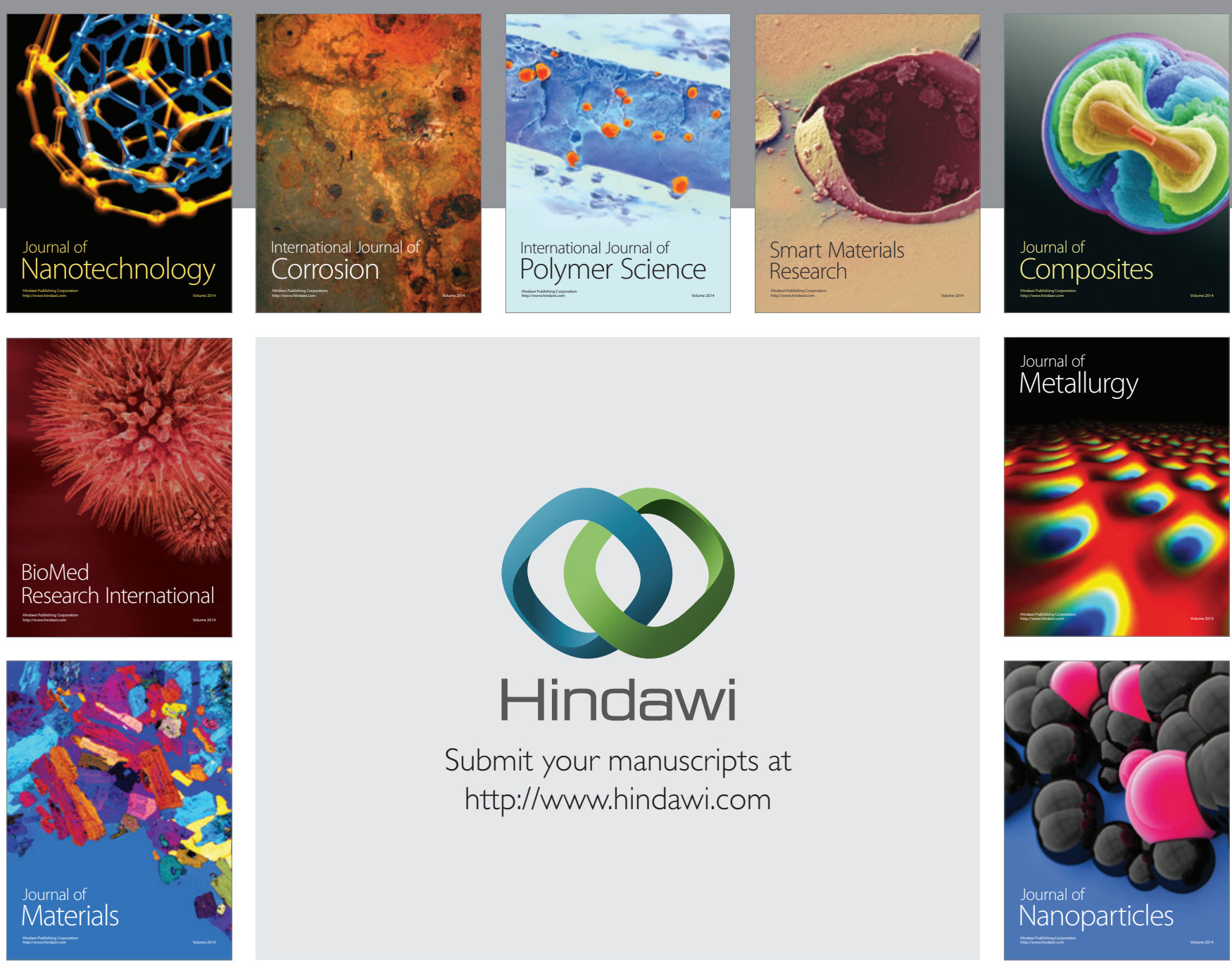

Submit your manuscripts at http://www.hindawi.com
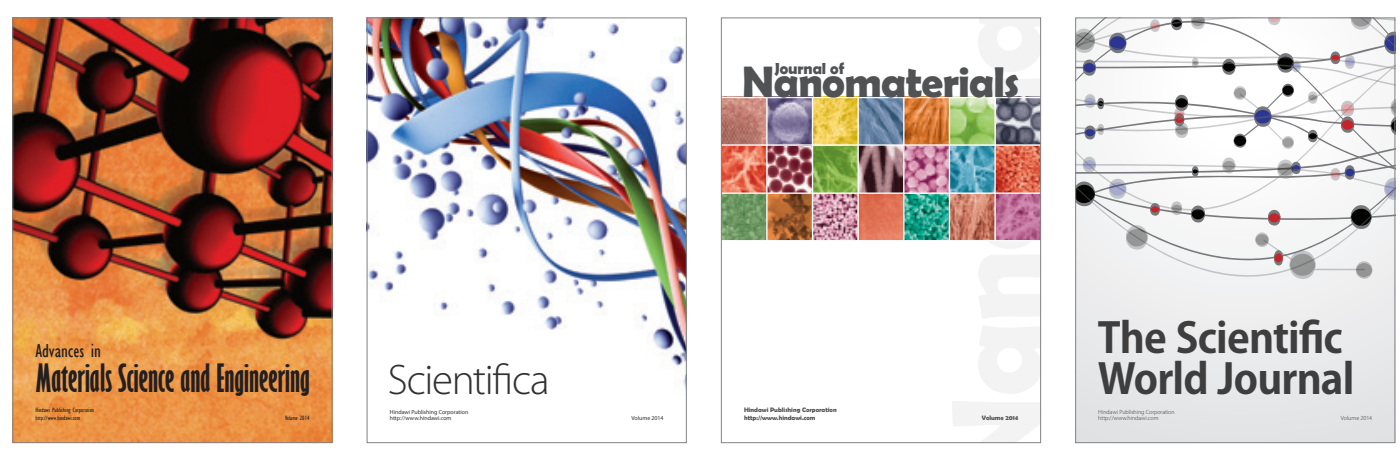

\section{The Scientific World Journal}
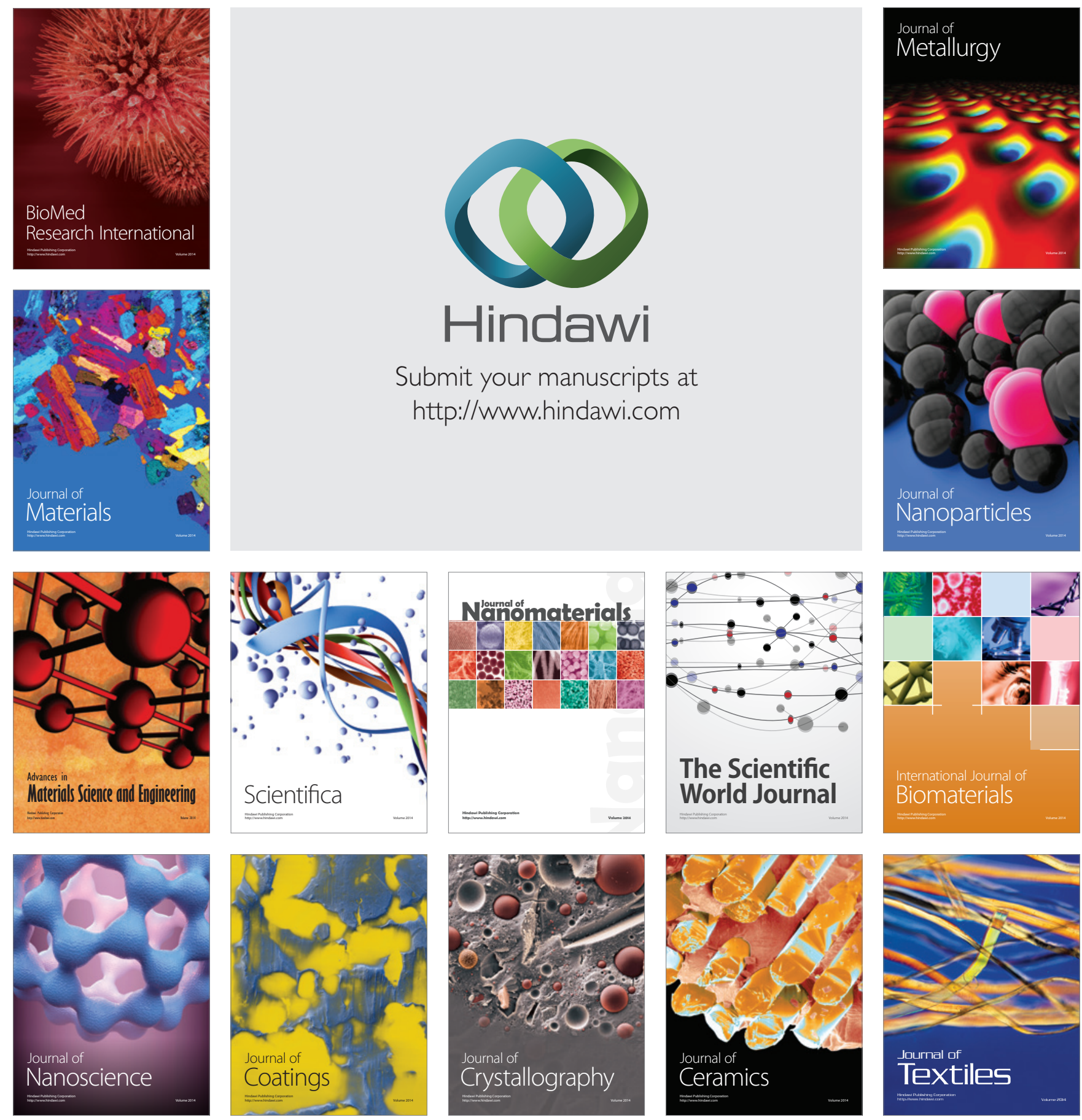